Bioscientia Medicina: Journal of Biomedicine \& Translational Research

Journal Homepage: www.bioscmed.com

\title{
Anatomy and Histologic of Intrinsic Aging Skin
}

\section{Putri Laksmi Karim ${ }^{*}$, Inda Astri Aryani ${ }^{1}$, Nopriyati ${ }^{1}$}

${ }^{1}$ Department of Dermatology and Venereology, Faculty of Medicine, Universitas Sriwijaya/ Dr. Mohammad Hoesin General Hospital, Palembang, Indonesia

\section{A R T I C L E I N F O}

\section{Keywords:}

Aging skin

Intrinsic aging

Histologic

*Corresponding author:

Putri Laksmi Karim

E-mail address:

putrilaksmikarim92@yahoo.com

All authors have reviewed and approved the final version of the manuscript.

https://doi.org/10.32539/bsm.v5i11.417

\section{A B S T R A C T}

Aging is an inevitable and dynamic biological process that is characterized by the progressive deterioration of body systems and declines in physiological reserve capacity. Aging skin has distinct two types: intrinsic and extrinsic. Intrinsic changes reduce collagen production, blood flow, amount of skin lipid, and loss of rete ridges. Intrinsic aging or chronological aging is cannot be restored to the skin with characterized by sagging skin and some expression of excess wrinkling lines. Intrinsic aging changes in thickness and characteristics of the epidermis, dermis, and hypodermis. Histologically, epidermis thinner by leveling off the dermoepidermal junction. In the dermis, collagen fibers become thicker and irregular than younger skin, reducing the elasticity of the skin, while hypodermis reduces lipid volume.

\section{Introduction}

The skin is a complex organ that covers the entire surface of the body. The skin provides protection as a physical barrier between the body and the environment, prevents water and electrolyte loss, reduces penetration of chemical exposure, and protects against pathogenic microorganisms. ${ }^{1}$ Aging is a process of biological change, dynamic and unavoidable characterized by a progressive deterioration of body systems and a decrease in a physiological reserve capacity. Skin is a part of the body that is in direct contact with the external environment and undergoes two types of skin aging, namely intrinsic and extrinsic aging. ${ }^{2}$

Intrinsic skin aging or chronological aging is a physiological change in aging skin and is influenced by genetic and hormonal factors. ${ }^{2}$ The theory of intrinsic skin aging with intracellular mechanisms is broadly divided into 3, namely telomere shortening, decreased Deoxyribonucleic (DNA) repair, and oxidative stress. ${ }^{3}$ Intrinsic aging is also influenced by several factors such as ethnicity, anatomical variations, and hormonal changes. ${ }^{4}$ Intrinsic aging includes decreased collagen production, decreased blood circulation, decreased fat content, and loss of rete ridges. This gives a clinical picture of dry, pale skin, fine wrinkles, lack of elasticity, and impaired skin repair. Intrinsic skin aging is also characterized by the development of various benign neoplasms, caused by impaired regulation of cellular proliferation. ${ }^{2}$ This literature review will discuss the mechanism of intrinsic aging based on 3 theories of intrinsic aging, intrinsic aging factors, as well as histological changes in the epidermis, dermis, and hypodermis/subcutaneous skin. 


\section{Intrinsic aging mechanism}

Intrinsic aging of the skin is characterized by sagging skin and some expression of excess frown lines. Changes in thickness and characteristics of the epidermis and dermis with aging can be seen in the schematic comparison of young and old skin (Figure
1).1,4 Intrinsically aging skin is less hydrated, less elastic, more permeable, and more prone to irritation due to decreased skin barrier function.2,5 The differences in the clinical and histologic features of intrinsic and extrinsic aging of the skin can be seen in Table 1.2

Table 1. Histological differences and clinical features of intrinsic and extrinsic aging ${ }^{2}$

\begin{tabular}{lll}
\hline & Intrinsic aging & Extrinsic aging \\
\hline Histological & Epidermal thinning & Solar elastosis \\
& $\begin{array}{l}\text { Lost rate ridges } \\
\text { Decrease in the amount of collagen } \\
\text { and elastin connective tissue }\end{array}$ & $\begin{array}{l}\text { Decrease in the number of fibroblasts } \\
\text { Decrease in the amount of extracellular } \\
\text { matrix }\end{array}$ \\
Xerosis & Xerosis \\
Pale & Multiple telangiectasia \\
& Slight wrinkles & Deep wrinkles \\
& Fecreased elasticity & Decreased elasticity \\
& & Fragility \\
& Dyspigmentation
\end{tabular}

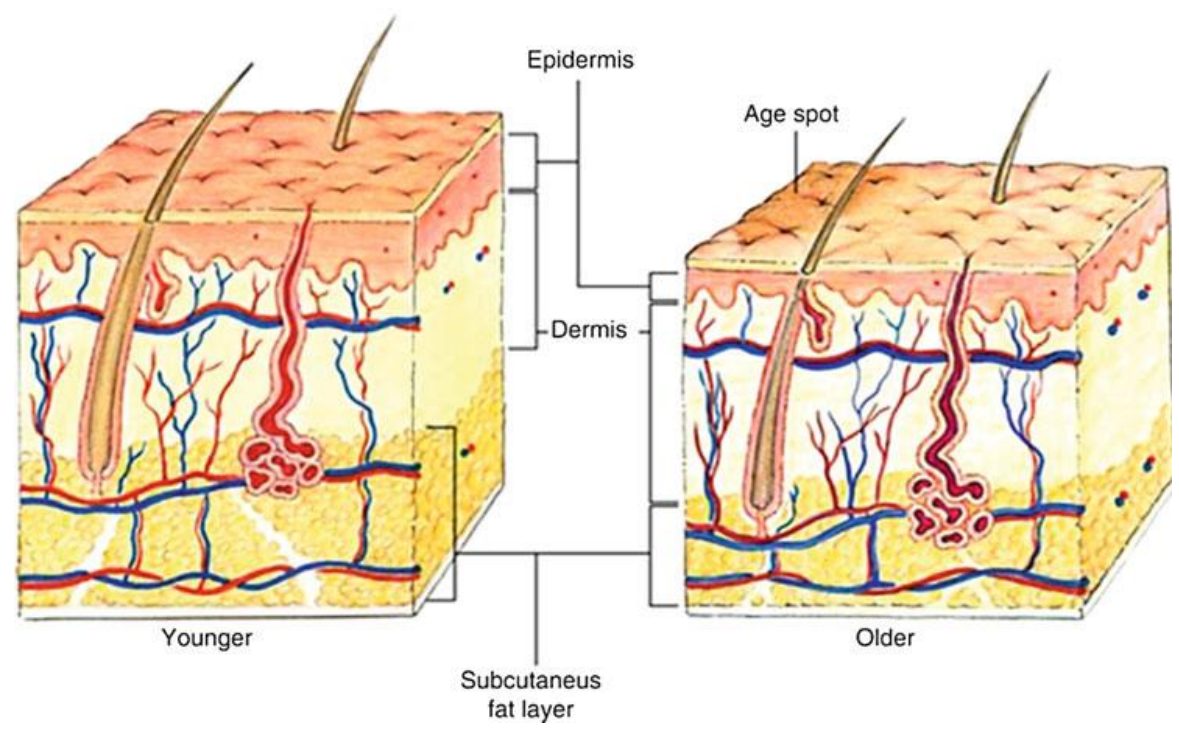

Figure 1. Differences in normal adult and aging skin structure. ${ }^{6}$

\section{Intrinsic aging theory}

The intrinsic aging theory is based on an outline of intracellular mechanisms divided into three, namely telomere shortening, decreased DNA repair, and oxidative stress.

\section{Telomere shortening}

Telomeres are repeated segments of DNA at the ends of chromosomes, consisting of several thousand base pairs. Telomere shortening is the result of the inability of DNA polymerase to replicate base pairs at the ends of chromosomes. ${ }^{2}$ Telomere length decreases with each cell division associated with physiological aging. Telomere shortening of keratinocytes, fibroblasts, and melanocytes in intrinsic aging averages 11-25 base pairs per year. The single-bond telomere overhangs form a loop structure that protects DNA integrity. Loop disruption will result in overhang segment damagecausing errors in coding and DNA damage responses including apoptosis and cellular senescence (Figure 2). $\cdot 2,3,7$ 
Telomeres shorten to a limit after proliferation causing cells to age and lose normal physiological functions. . $^{2,3,8}$

Kosmadaki and Gilchrest described the final the telomere end. Repeated pair 3'TTAGGG overhang activates p53 signaling resulting in the final process of proliferative aging and apoptosis. $2,8,9$

pathway for intrinsic senescence and photoaging

leading to disruption of the normal loop structure at

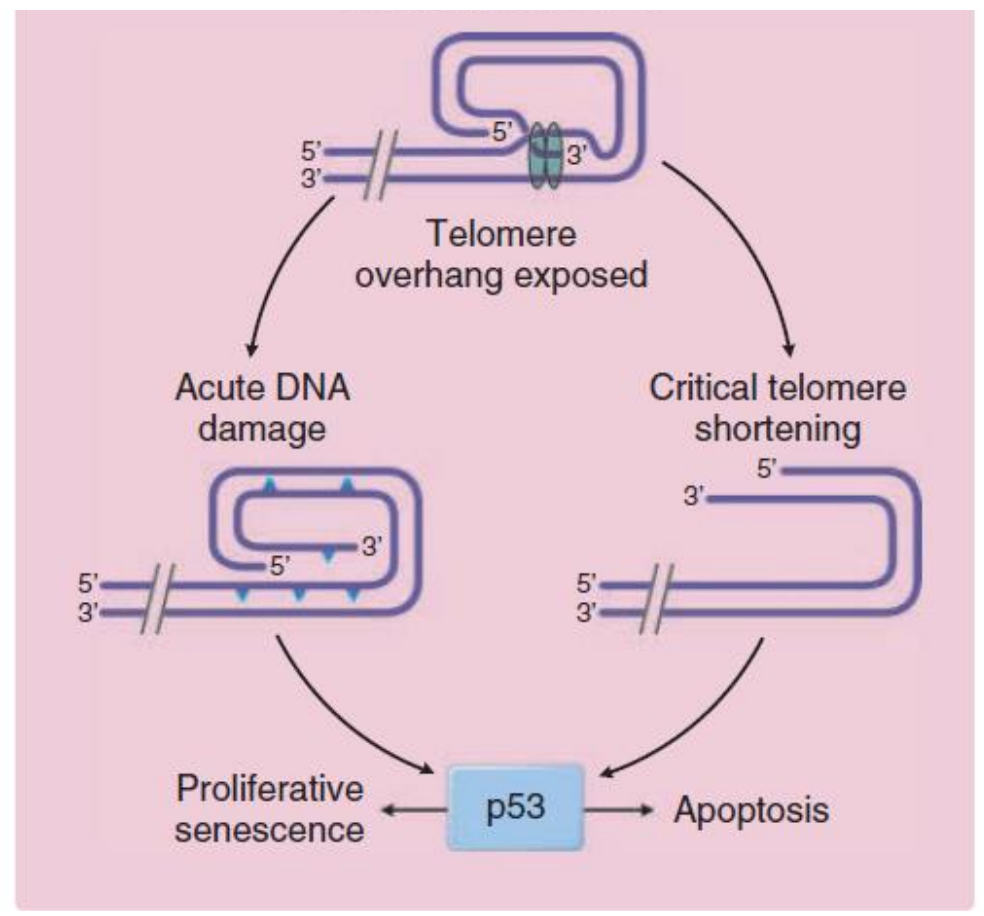

Figure 2. Schematic of the cellular response of telomere shortening triggers. ${ }^{2}$

\section{Decreased DNA repair}

Cells have DNA repair mechanisms to remove damaged segments. Cells process DNA repair mechanisms to remove segmental damage mainly through nucleotide excision repair or excision repair pathways during normal growth and metabolic phases (G1) and growth and mitotic preparation phases (G2). Cells perform apoptosis to protect the organism from cancerous changes. ${ }^{2}$ A significant decrease in the synthesis phase (S) of skin fibroblast cells in old age, indicates that the potential for cellular replication is impaired in fibroblast cells. Decreased DNA repair leads to accumulation of DNA damage in aged skin and results in chromosomal instability, cell growth arrest, and apoptosis, as well as chronic skin inflammation by oxidative stress. ${ }^{2,3,7}$

\section{Oxidative stress}

Oxidative stress affects the intrinsic aging process. Free radicals are generated endogenously and exogenously causing oxidative damage to cellular components including DNA. ${ }^{3}$ The complex antioxidant system of enzymatic and nonenzymatic effectors evolves to scavenge endogenous and exogenous free radicals in the skin.2,3 Antioxidants function to maintain normal cell function, both non-enzymatic antioxidants such as glutathione and enzymatic antioxidants such as superoxide dismutase, catalase, and glutathione peroxide. ${ }^{2}$ Oxidative damage to cellular proteins and age-related decrease in proteasome activity result in accumulation of protein damage and activation of active Oxygen Species (ROS) thereby interfering with normal cellular function. ${ }^{2,3,7}$ 


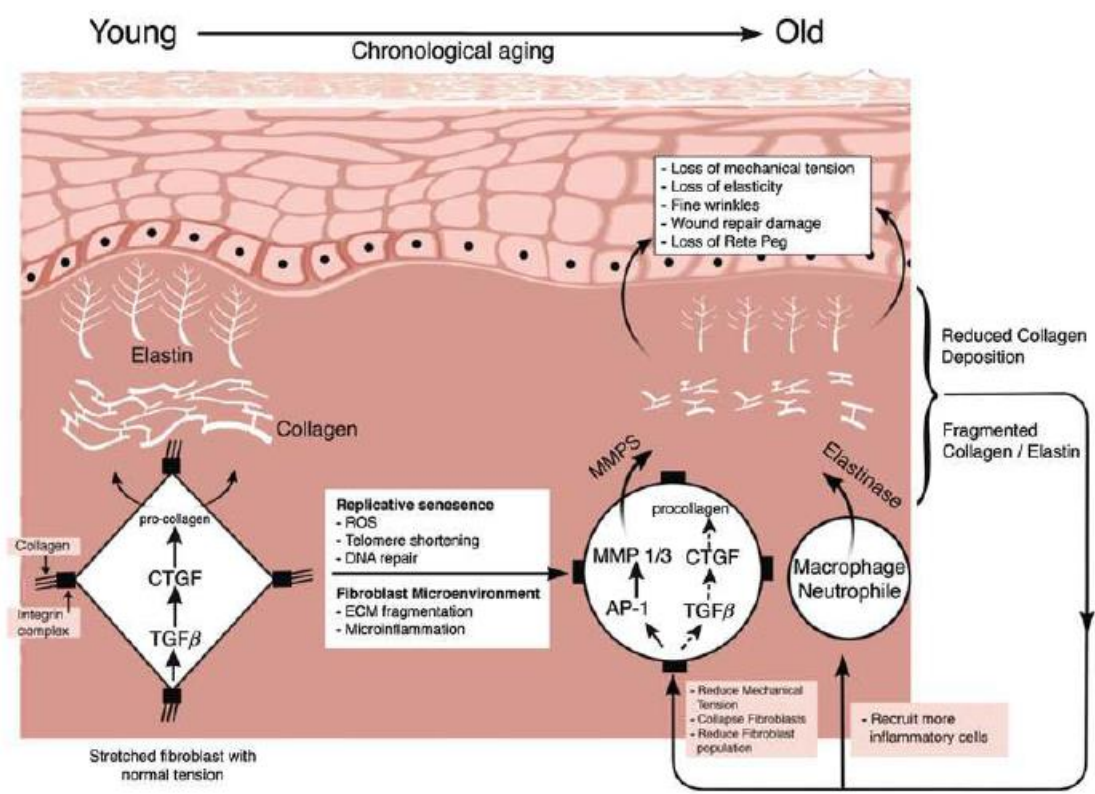

Figure 3. Intrinsic skin aging changes. ${ }^{3}$

\section{Intrinsic skin aging factors}

The physiological changes of skin in intrinsic skin aging are influenced by several factors, including ethnic factors, anatomical variation factors, and hormonal factors. 4

\section{Ethnic factor}

Ethnic factors relate to skin type and the amount of skin melanin/pigmentation. Pigmentation is the biggest effect of skin aging, high levels of pigmentation are protective against the cumulative effects of photoaging. ${ }^{4}$ African-American skin is denser than Caucasian skin and has a higher cellular lipid content, making it more resistant to aging. Asian ethnic wrinkles occur more slowly with lower severity than Caucasians. 4,10

\section{Anatomical variation factor}

Anatomical variation factors consist of thickness, skin stiffness, and lipid composition. The decrease in epidermal thickness was smaller at the temples than at the volar forearms in old age. The lipid composition of the stratum corneum layer varies, there is an inverse relationship between the percentage of lipids in certain body parts and the ability of permeability. Skin stiffness is higher on the forehead than on the cheeks in post-menopausal women. The decline in sensory perception with aging is more pronounced in the nasolabial folds and cheeks than in the chin and forehead. 4

\section{Factors hormonal changes in skin tissue}

Estrogen affects skin collagen, dermis thickness, elasticity, and water content for skin protection. Estrogen triggers epidermal mitotic activity, but the exact mechanism is not known. ${ }^{3}$ On the epidermis, $17 \beta$-estradiol stimulates the proliferation and synthesis of keratinocyte DNA, estradiol stimulates cell proliferation through cell membrane receptors. ${ }^{11 E}$ strogen plays a protective role in the human epidermis using estradiol preventing apoptosis induced by hydrogen peroxide in epidermal keratinocytes. Estrogen in the dermis affects skin thickness by stimulating the synthesis, maturation, and turnover of collagen (Table 2).2,7 Estrogen is also believed to protect photo-aging. Skin fibroblasts increase collagen synthesis by $76 \%$ in response to estrogen. ${ }^{7,11}$ 
Skin components

Epidermis

Dermis

Gland

Hair follicles

\section{Effect of estrogen}

Increases mitotic activity of epidermal cells

Epidermal melanocyte stimulation

Increases collagen synthesis, maturation, and turnover

Improves the morphology of collagen and elastic fibers

\section{Normal skin histologic and intrinsic aging}

Normal skin structure varies depending on the skin region. Differences in skin structure depend on the thickness of the layers of the epidermis, dermis,
Reduces the activity of the sebaceous glands and

Apocrine

Affects the hair follicle cycle Inhibits follicular melanocyte activity

Table 3. Histological features of aging human skin ${ }^{6}$

\begin{tabular}{ll}
\hline Skin components & \multicolumn{1}{c}{ Aging effect } \\
\hline \multirow{3}{*}{ Epidermis } & Lower lipid content \\
& The dermo-epidermal junction is thinned \\
& The number of active melanocyte enzymes decreases by 8-20\% per decade \\
& Langerhans cell count is reduced \\
& Reduced capacity for re-epithelialization \\
& The number of skin pores increases \\
& Decreased thickness (atrophy) \\
& Decreased vascularity and cellularity \\
Dermis & Decreased collagen synthesis \\
& Pacinian and Meissner's weight loss \\
& The structure of the sweat glands becomes distorted, the number of functional \\
& sweat glands decreases \\
& Degradable elastic fiber \\
& Decreased number of blood vessels \\
& Decreased number of nerve endings \\
& Changes in subcutaneous fat distribution \\
& Overall volume reduced \\
& Hair loses normal melanin \\
Hair thinning \\
Decreased number of sweat glands \\
The nail plate becomes abnormal \\
Reduced sebum production \\
\end{tabular}

\section{Epidermis}

The epidermis layer is composed of stratified squamous epithelial cells that undergo continuous renewal. Keratinocytes gradually move from the basement membrane of the skin to the surface of the skin, forming several layers of the epidermis, namely: stratum basale or stratum germinativum, stratum spinosum, stratum granulosum, and stratum corneum (Figure 4). 3,4 An additional layer on the palms of the hands and soles of the feet, the stratum lucidum, lies between the stratum corneum and stratum hypodermis, and melanocyte content. ${ }^{1}$ Changes in skin structure in aging can be seen in Table 3. 
basal layer cells become less uniform, although the mean cellular size increases. ${ }^{6}$ Histological changes at the dermo-epidermal junction include flattening and loss of the rete ridges ${ }^{2,5}$

The thickness of the skin increases during the first 20 years of life (although the number of cell layers remains stable) and begins to thin progressively with age in all layers of the skin.4,6 Epidermal skin area thins by $30-50 \%$ between the ages of 30 to 80 years with age. ${ }^{2,3}$ The decrease in epidermal thickness is most pronounced in exposed areas, such as the face, neck, upper chest, and areas of the extension surfaces of the hands and forearms. 2,6,12 Overall, the thickness of the epidermis decreases by about $6.4 \%$ per decade, decreasing more rapidly in women than in men. ${ }^{4.6}$ The wound healing process is slower and desquamation is less effective due to decreased epidermal cell turnover. ${ }^{13,14}$

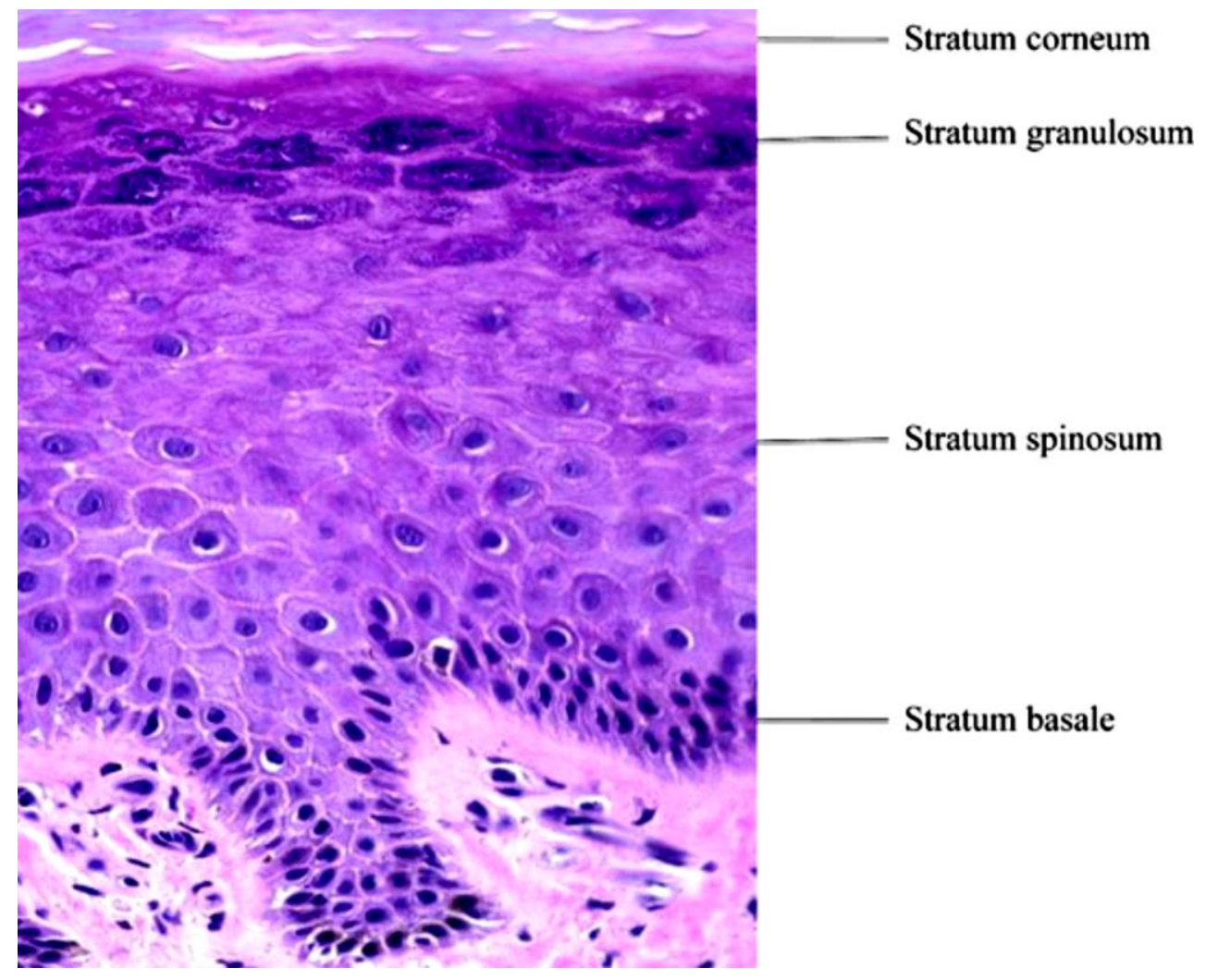

Figure 4. Epidermis layer (hematoxylin-eosin, magnification 40x). ${ }^{3}$

The skin barrier is damaged in aging. This is due to a decrease in stratum corneum lipids, a reduction in the loss of the lamellar bilayer in the stratum corneum gap. Epidermal filaggrin decreases with age, resulting in impaired skin protection function resulting in dry and scaly skin. ${ }^{2}$ The integrity of the stratum corneum skin barrier depends on the regulation of lipid composition. The total lipid content of old age skin is reduced by $65 \%$. Ceramide levels are significantly reduced in aging skin. Triglycerides are reduced due to the breakdown of stratum corneum lipids. ${ }^{6}$

The water content of the stratum corneum is lower than that of young skin. The amount of natural moisturizing factor (NMF) of the skin is reduced, thereby reducing its water-binding capacity. The water content of the stratum corneum decreases gradually below normal levels causing desquamation disorders. Desquamation causes keratinocytes to accumulate and adhere to the skin's surface, resulting in rough, scaly, and pigmented skin in aging skin. ${ }^{2,6}$ 


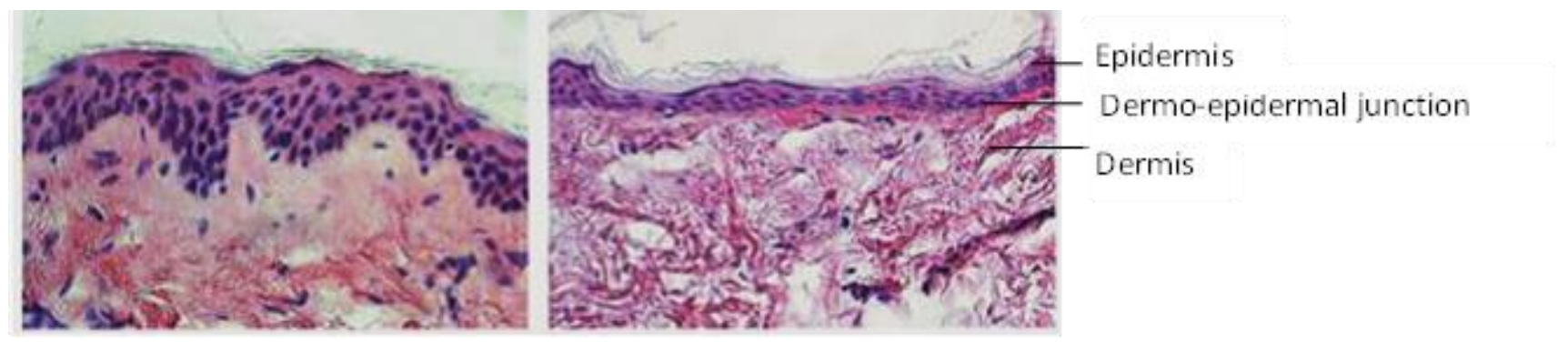

Figure 5. Histological differences between young skin (A. 28 years) and skin on intrinsic aging (B. 69 years). Common signs of skin aging are seen in B, irregular extracellular matrix, thinned epidermis consisting of only a few layers, flat dermo-epidermal junction with no papillary dermis structure.

Glycosaminoglycans (GAGs) in the epidermis have decreased in number. Hyaluronic acid (HAs) is the major type of GAGs and produces fibroblasts in epidermal and dermal keratinocytes. The decrease in the number of HAs in the epidermal intracellular space in the spinosum layer causes a decrease in water binding and some changes in aging skin such as wrinkling, changes in elasticity, decreased turgidity, and decreased microvascular support capacity. ${ }^{3}$ Characteristics of aging human skin are illustrated in histological images of breast skin biopsies of young (19 years old) and old (74 years old) volunteers (Figs 6A and 6B). In general, the epidermis is thicker at a young age than in old age. The structure of the reteridges is more clearly visible on the skin of young ages,

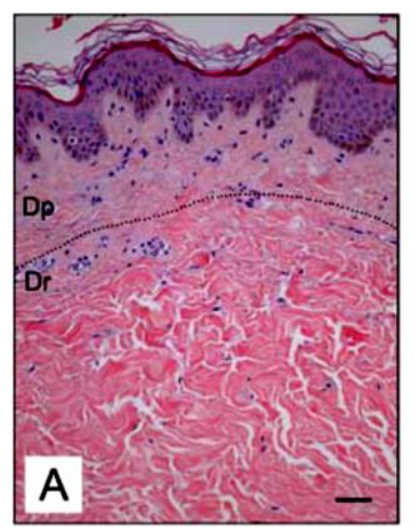

whereas the skin of old age is characterized by flattening of the dermo-epidermal junction. ${ }^{13}$

\section{Melanocytes}

Melanocytes originate from neural crest cells and migrate to the epidermis, where melanin is produced.1,15Ethnic variations in pigmentation are due to differences in melanocyte activity, not differences in melanocyte numbers. Melanocyte density decreases by $6 \%$ to $8 \%$ per decade with age, melanocyte density is higher in sun-exposed skin than in unexposed skin. ${ }^{1}$ The enzymatic activity of melanocytes decreases by 8$20 \%$ per decade, resulting in uneven pigmentation in aged skin and an increased risk of skin cancer.6,8

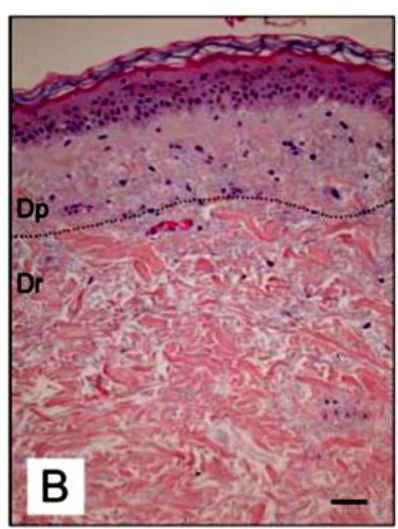

Figure 6. Histological characteristics of human skin; young people and old people. The photos shown correspond to histological sections of breast skin biopsies obtained from 19 years old (A) and 74 years old donors (B). Sections were stained with hematoxylin, eosin, and saffron (HES). Scale bar $=50 \mathrm{~mm} . \mathrm{Dp}=$ papillary dermis. $\mathrm{Dr}=$ reticular dermis. ${ }^{13}$

\section{Langerhan cells}

Langerhans cells are antigen-presenting cells derived from the bone marrow. Langerhans cells make up 3\%-6\% of the epidermis, mainly in the spinosum layer. The aging process and chronic sun exposure cause the number of Langerhans cells to decrease. ${ }^{1}$
Langerhans cell is reduced in number in the epidermis, experiencing structure change, have fewer and shorter dendrites, resulting in weakened skin defense mechanisms and decreased antigen percentage ability in old age.2,6 Table 3 summarizes the cellular changes in aging skin in the epidermis. 
Table 3. Changes in aging-related to epidermal cell type ${ }^{2}$

\begin{tabular}{|c|c|c|}
\hline Cell type & Structural changes & Function change \\
\hline Epidermal Keratinocyte & Epidermal dyscrasia & $\begin{array}{l}\text { Reduces mitotic activity } \\
\text { Improved migration time } \\
\text { Improved aging }\end{array}$ \\
\hline Melanocytes & $\begin{array}{l}\text { Atypia } \\
\text { Decrease in quantity } \\
\text { Functional melanocytes } \\
\text { An increased amount of } \\
\text { photodamaged in skin }\end{array}$ & Decreased melanin production \\
\hline Langerhan's cells & $\begin{array}{l}\text { The slight decrease in quantity } \\
\text { and shorter dendrites }\end{array}$ & $\begin{array}{l}\text { Decreasing ability antigen } \\
\text { presentation }\end{array}$ \\
\hline
\end{tabular}

\section{Dermo-epidermal junction}

The dermo-epidermal junction is a basement membrane synthesized by basal keratinocytes and dermal fibroblasts (Figure 7). The function of the dermo-epidermal junction is as a support for the adhesion of the dermis to the epidermis and a chemical barrier to the skin. The structure of the dermoepidermal junction consists of 4 distinct layers, visible through an electron microscope. ${ }^{1}$

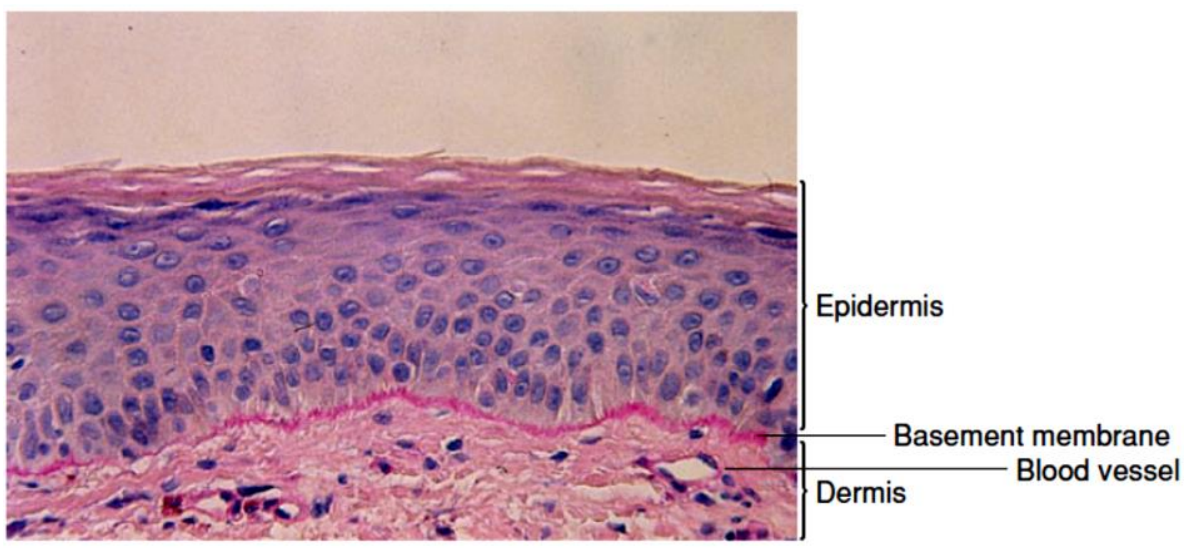

Figure 7. Histologic of the dermo-epidermal junction. Basal membrane dividing the epidermis and dermis. ${ }^{16}$

Structural changes in the form of flattening of the dermo-epidermal junction, occur due to a decrease in the number and size of dermal papillae. ${ }^{4,8}$ Histological picture shows a reduced number of papillae, from an average of 40 papilla $/ \mathrm{mm}^{2}$ in young skin to 14 papilla/mm2 in people over 65 years of age.4,6 Flattening of the dermo-epidermal junction results in a thinner epidermis due to retraction of the rete ridges, reducing the thickness of the dermo-epidermal junction by $35 \%$. Depletion of the dermo-epidermal junction and papilla are reduced leading to increased skin fragility and reduced nutrient transfer between the dermis-epidermis, the skin becomes vulnerable to friction and easily damaged.6,13 This flattening can impair basal cell proliferation and may affect percutaneous absorption. Flattening of the dermoepidermal junction may also contribute to wrinkle formation by increasing the susceptibility to dermoepidermal separation. 6,5

\section{Dermis}

The dermis is divided into two parts: papillary and reticular. The papillary dermis lies below the dermoepidermal junction and is known as the dermal papilla, interspersed with the epidermal rete ridge. Epidermal rete ridges increase the junction area between the dermis and epidermis and allow for better adhesion between the dermis and epidermis. The papillary dermis is composed of loose collagen bundles, elastic fibers, fibrocytes, blood vessels, and nerve endings. The 
reticular dermis contains compact collagen fibers, and the elastic fibers are thicker. ${ }^{1}$ The total thickness of the skin in old age decreases due to the loss of collagen and elastin in the dermis. Postmenopausal women experience a decrease in skin thickness of $1.13 \%$ per year, equivalent to a decrease in the collagen content of $2 \%$ per year. Skin thickness, vascularity, and cellularity also decrease with age.2,6,8

Collagen is the main constituent of the dermis and is made of fibroblast collagen fibers. Interstitial collagen makes up more than $90 \%$ of skin fibers, especially types I and III, which provide tensile strength and mechanical resistance to the skin. The dermis becomes atrophic with a reduced number of fibroblasts in aging. 2,3,6 Changes in the dermis can be seen in Table 4.

Table 4. changes in aging-related to dermal components ${ }^{2}$

\begin{tabular}{lll}
\hline Dermal components & Structural changes & Function change \\
\hline Collagen & $\begin{array}{l}\text { Increased stiffness } \\
\text { Reduction of type I and collagen }\end{array}$ & $\begin{array}{l}\text { Wound healing disorders } \\
\text { Reduction of mechanical stress } \\
\text { on fibroblasts ( } \rightarrow \text { decrease } \\
\text { collagen synthesis) }\end{array}$ \\
Improved fragmentation & Reduce the number and \\
Elastin & $\begin{array}{l}\text { elastic fiber diameter } \\
\text { solar elastosis (photodamage) }\end{array}$ & \\
& & \\
Basic substance & $\begin{array}{l}\text { Abnormal deposition of } \\
\text { glycosaminoglycans prevents } \\
\text { normal interactions with water } \\
\text { Reduce hyaluronic acid }\end{array}$ & Decreased skin hydration \\
& & \\
& & \\
\end{tabular}

Aging is associated with decreased collagen turnover due to decreased fibroblasts and decreased collagen synthesis. The proportion of collagen type I to collagen III in young skin is in the 6:1 ratio, the ratio decreases significantly with age, where type I collagen is selectively lost (Figs. 8a and b). The collagen fibers of the old age dermis become thick bundles and the collagen becomes irregular compared to that of young skin. ${ }^{2,3}$ Overall collagen content decreases by about $1 \%$ per year per body surface area. The loss of collagen in intrinsic aging leads to epidermal and dermal atrophy, with flattening of the rete ridges and formation of wrinkles. ${ }^{3}$ Collagen cross-links stabilize and reduce skin elasticity. ${ }^{2}$
Elastin is a protein in connective tissue, which functions to restore the shape of the tissue to its original shape after stretching or contraction. Elastin function decreases with aging, elastin calcifies and elastin fibers degrade, and elastin turnover decreases. (Figure 8c).2,6 Loss of the structural integrity of the dermis leads to increased stiffness, decreased stretch ability, and reduced elasticity, these processes occurring more rapidly in women than in men. The impact of these changes is seen when the skin is subjected to mechanical stress, recovery occurs within minutes in young skin, but takes more than 24 hours in old skin. ${ }^{3,17}$ 


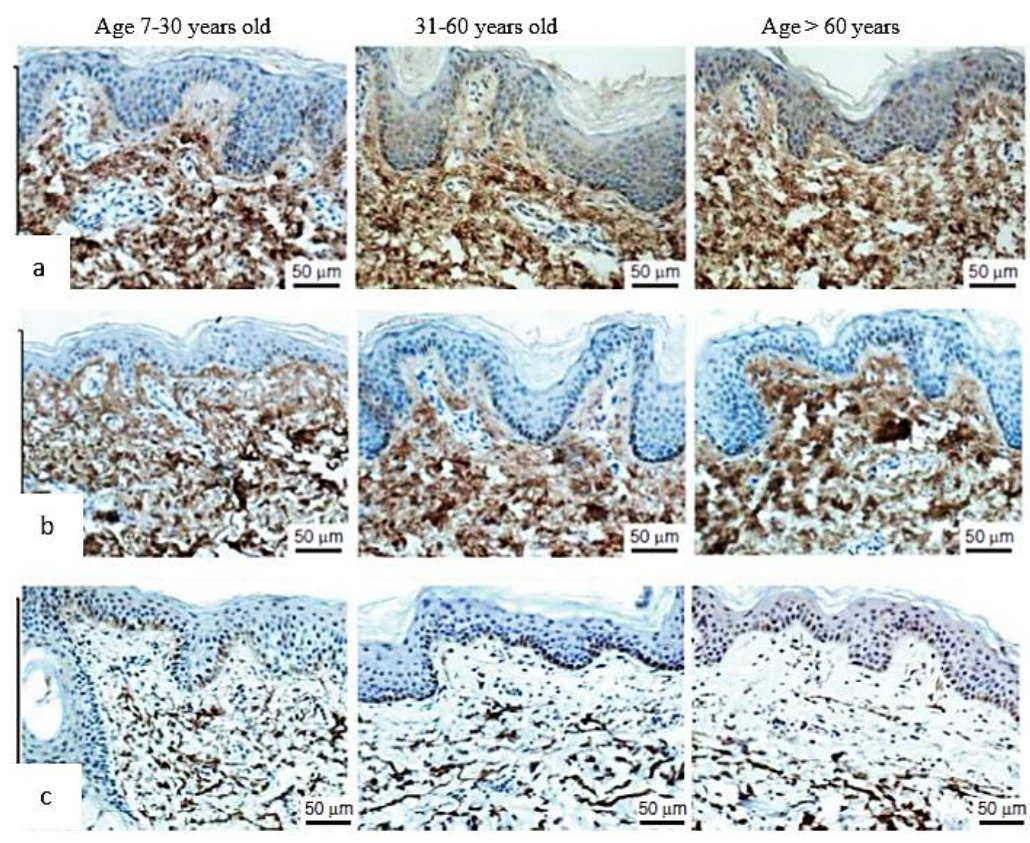

Figure 8. Histologic changes of skin in aging with histochemical staining: collagen type I (a), collagen type III(b), elastin (c) are thicker at a young age and decrease with age. ${ }^{8}$

\section{Blood vessel}

The skin has many blood vessels, it consists of 2 tissues arranged horizontally: the superficial and the deep part of the subdermal plexus. This tissue originates from the perforating arteries below the muscle and communicates via small blood vessels, crossing the dermis vertically. The subdermal plexus is located near the dermal-hypodermal junction and supplies hair follicles and sweat glands. ${ }^{1}$ Endothelial cell are enveloped by a basement membrane containing enmeshed pericytes (EC) and pericytes cells (PC). The density of skin micro-vessels depends on age and reduced capillary function.6,18 With aging, the density of capillaries and venules decreases in the plexus and pericyte loss occurs during the aging process. Histologic of skin blood vessels can be seen in figure 9.18 Decreased vascular tissue in aging skin causes decreased blood flow, impaired nutrient turnover, inhibited thermoregulation, and lowered skin surface temperature. ${ }^{3}$

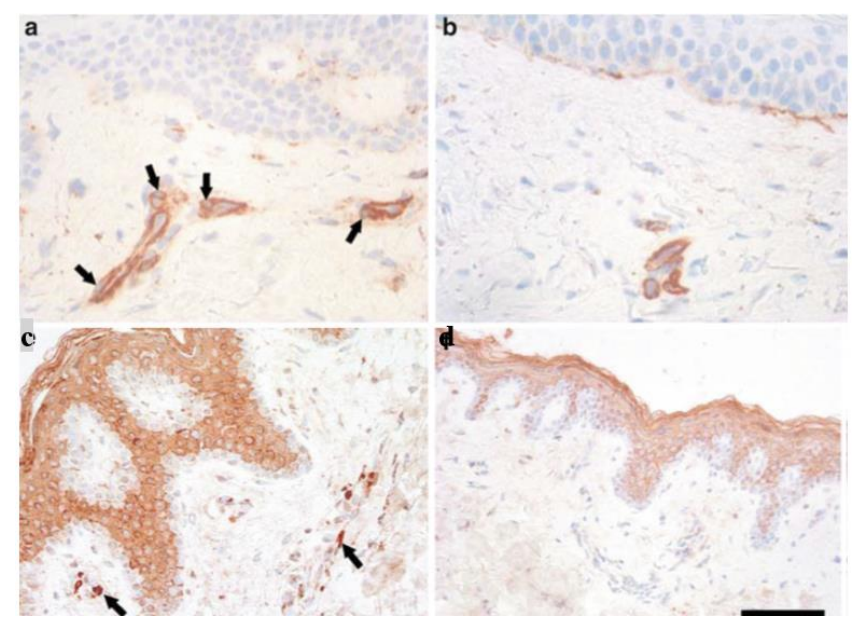

Figure 9. Histological blood vessels in aging. Microvascular density chart by age: Left side (a, c,) young age skin; Right side (b, d,) old age. ${ }^{18}$ 


\section{Hypodermic}

The hypodermis is the deepest layer of skin, located below the dermis and above the muscles. The hypodermis protects the body from mechanical injury, as well as serves as a supply of energy reserves. The main cells of the hypodermis are fat arranged in lobules separated by connective tissue septa. The thickness of this layer shows variations in individual anatomic conditions and reflects individual nutritional status. ${ }^{1}$ The hypodermis loses much of its fat pad as humans age. ${ }^{6}$ The overall volume of subcutaneous fat usually decreases with age by up to $30 \%$, although the overall proportion of subcutaneous fat throughout the body increases until the age of 70 years. Fat in the face, hands, and feet was reduced, while increased in the thighs, waist, and abdomen due to changes in fat distribution. ${ }^{3,6}$

\section{Skin adnexa}

Skin adnexa is specialized epithelial structures located mainly in the dermis and hypodermis, and are connected to the epidermis. The skin adnexa consist of pilosebaceous units and sweat glands. The adnexa play an important role in the epithelialization phase of wound healing. ${ }^{1}$
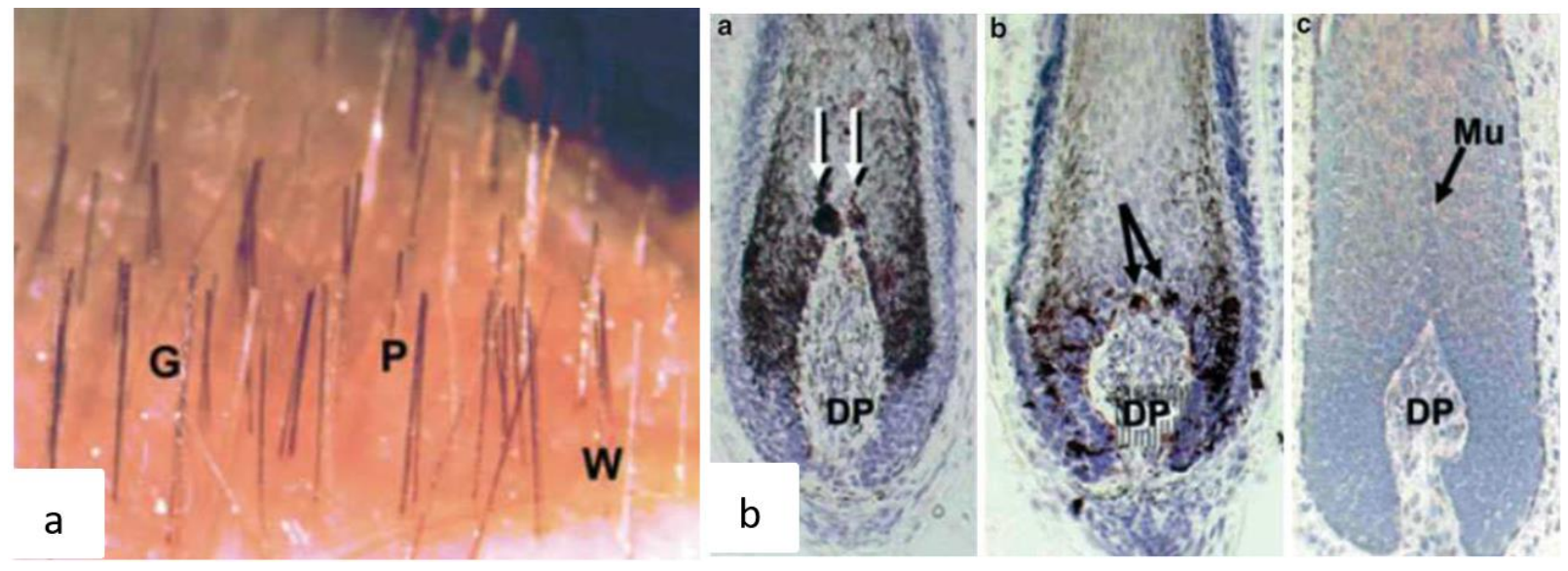

Figure 11. Hair color change (a): gray $(\mathrm{G})$, White $(\mathrm{W})$, black/pigmented (P). Histological features of hair melanocyte loss (b): follicular dermal papilla (DP), medulla (Mu) ${ }^{3}$

\section{Sweat glands}

Sebaceous glands are found in most parts of the body except the palms of the hands and soles of the feet, mostly on the face. Sebaceous gland size increases

\section{Pilosebaceous unit}

The pilosebaceous unit consists of hair, hair follicles, sebaceous glands, and erector pili muscles, found throughout the integument, except on the palms of the hands, soles of the feet, and some of the genitals. ${ }^{6}$ The hair follicle consists of several segments: the infundibulum, isthmus, lower follicle, and hair bulb. The rate of hair growth decreases and the thickness of the hair decreases with age. The hair bulb contains hair matrix cells and melanocytes that function in hair pigmentation. ${ }^{1,2,6}$

The hair cycle consists of three main phases of growth (anagen), regression (catagen), and resting (telogen), with melanogenesis occurring in the anagen phase. Each new hair cycle is associated with the formation of the pigment unit of the hair follicle. ${ }^{6}$ The aging process and changes in hair color occur around the age of 35-40 years or about ten hair cycles. Active melanin production decreases with each new hair cycle leading to reduced melanin (grey hair) or no melanin deposition (white hair) after the first ten hair cycles. ${ }^{3,6}$ The decrease in the number of bulbar melanocytes is one of the reasons for the decrease in melanin production in aging. ${ }^{6,15}$ 
are the main sweat glands in humans and play an important role in the process of thermoregulation are found in almost all parts of the skin except the vermilion border of the lips and nail beds, which have a maximum density on the palms, soles, axillae, and forehead. ${ }^{1}$ With aging, the number and function of the eccrine sweat glands decrease. ${ }^{2,6}$

\section{Conclusion}

Intrinsic aging is a physiological change in the skin, including decreased collagen production, decreased blood circulation, decreased fat content, and loss of rete ridges. The intrinsic aging theory includes telomere shortening, decreased DNA reparability, and oxidative stress. Several factors play a role in intrinsic aging such as ethnicity, anatomical variations, and hormonal changes.

Histological changes in aging skin are seen in the epidermis, dermis, hypodermis, and adnexa of the skin. In the epidermis, there is a thinning of the dermoepidermal junction, a decrease in the number of melanocytes and Langerhans cells. The dermis in aging undergoes a decrease in thickness, synthesis of collagen and elastin fibers, and a decrease in the number of blood vessels. Changes in distribution and a decrease in fat volume occur in the hypodermis layer. In the adnexa, there is a decrease in melanin, the number of sweat glands, and sebum production.

\section{References}

1. Khavkin J, Ellis DAF. Aging skin: histology, physiology, and pathology. Facial Plast Surg Clin NA. 2011;19(2): 229-34.

2. Kerns ML, Chien AL. aging skin. In: Sewon K, Masayuki A, editors. Fitzpatrick's Dermatology. 9th ed. New York: McGraw-Hill Education; 2019; 1779-91.

3. Jia Q, Nash JF. Pathology of aging skin. In: Miranda A. Farage KWM, Maibach HI, editors. Textbook of Aging Skin. 2nd ed. Berlin Heidelberg: Springer-Verlag; 2017; 363-85.

4. Farage MA, Miller KW, Elsner P, Maibach HI. Intrinsic and extrinsic factors in skin aging: a review. Int J Cosmetic Sci. 2011; 30: 87-95.
5. Tobin DJ. Introduction to skin aging. J Tissue Viability. 2017; 26(1): 37-46.

6. Farage MA, Miller KW, Maibach HI. Degenerative changes in aging skin. In: Miranda A. Farage KWM, Maibach HI, editors. Textbook of Aging Skin. 2nd ed. Berlin Heidelberg: Springer-Verlag; 2017; 15-30.

7. Makrantonaki E, Zouboulis CC. Pathomechanisms of endogenously aged skin. In: Miranda A. Farage KWM, Maibach HI, editors. Textbook of Aging Skin. 2nd ed. Berlin Heidelberg: Springer-Verlag; 2017; 111-20.

8. El-domyati M, Medhat W. Skin aging: an immunohistochemical evaluation. In: Miranda A. Farage KWM, Maibach HI, editors. Textbook of Aging Skin. 2nd ed. Berlin Heidelberg: Springer-Verlag; 2017; 469-84.

9. Kosmadaki MG, Gilchrest BA. The role of telomeres in skin aging/photoaging. Micron. 2004; 35: 155-9.

10. Hillebrand G. Facial wrinkling: the marquee clinical sign of aging skin. In: Miranda A. Farage KWM, Maibach HI, editors. Textbook of Aging Skin. 2nd ed. Berlin Heidelberg: Springer-Verlag; 2017; 1055-64.

11. Thornton MJ. Estrogens and aging skin. Dermatoendocrinol. 2013; 5(2): 264-70.

12. Assaf H, Adly MA, Hussein MR. Aging and intrinsic aging: pathogenesis and manifestations. In: Miranda A. Farage KWM, Maibach HI, editors. Textbook of Aging Skin. 2nd ed. Berlin Heidelberg: Springer-Verlag; 2017; 783-94.

13. Fortunel NO, Asselineau D. Aging alters functionally human dermal papillary fibroblast but not reticular fibroblast: a new view of skin morphogenesis and aging. PLOS One. 2008; 3(12).

14. Millsop JW, Chang ALS. Major changes in skin function in the elderly and their contributions to common clinical challenges. In: Miranda A. Farage KWM, Maibach HI, editors. Textbook of Aging Skin. 2nd ed. Berlin Heidelberg: Springer-Verlag; 2017; 43-52. 
15. Cichorek M, Wachulska M, Stasiewicz A, Tymińska A. Skin melanocytes: biology and development. Derm Allergol. 2013; 30-41.

16. Baumann L. Basic science of the dermis. In: Leslie Baumann, editor. Cosmetic Dermatology: Principles and Practice. 2nd ed. New York: The McGraw-Hill Companies, Inc; 2009; 8-13.

17. Bhattacharyya TK. An overview of the histology of aging skin in laboratory models. In: Miranda A. Farage KWM, Maibach HI, editors. Textbook of Aging Skin. 2nd ed. Berlin Heidelberg: Springer-Verlag; 2017; 37-41.

18. Helmbold P. Histology of microvascular aging of human skin. In: Miranda A. Farage KWM, Maibach HI, editors. Textbook of Aging Skin. 2nd ed. Berlin Heidelberg: Springer-Verlag; 2017; 3-10. 